\title{
Pregnancy-associated spontaneous coronary artery dissection (PASCAD): An etiology for chest pain in the young peripartum patient
}

\author{
Richard Lee, BHSc, MD*; David Carr, MD, CAC, CCFP(EM) ${ }^{\dagger \ddagger \S}$
}

\begin{abstract}
Cardiac emergencies in pregnancy and the postpartum period are rare but often life-threatening. An emergency physician's differential diagnosis for chest pain in the peripartum patient often includes serious etiologies such as pulmonary embolism or myocardial infarction (MI). A lesser-known but important consideration on the differential for $\mathrm{MI}$ is that of a spontaneous coronary artery dissection (SCAD). SCAD is defined as an intramural hematoma within the coronary artery that compresses the true lumen. Expansion by increased pressures may lead to subsequent myocardial ischemia and infarction. This condition is the most common cause of pregnancy-associated MI and is reported as the cause of MI in $24 \%$ to $35 \%$ of all women younger than 50 years. This condition is predominately seen in young healthy females with no traditional risk factors for coronary artery or cardiac disease, and typically in the postpartum period. SCAD in the peripartum period is defined as pregnancy-associated spontaneous coronary artery dissection (PASCAD). Abnormal ECG changes, elevated troponins, and regional wall motional abnormalities on echocardiography are all diagnostic findings of SCAD, which can be ultimately confirmed with coronary angiography. Failure to immediately address this condition can lead to acute heart failure, cardiogenic shock, and death. Thrombolytic treatment may be harmful and is not recommended, and percutaneous coronary intervention can result in the iatrogenic propagation of further coronary dissection. As a result, the management for suspected SCAD involves emphasis on urgent transfer and urgent coronary artery angiography to determine appropriate treatment modalities.
\end{abstract}

\section{RÉSUMÉ}

Les urgences cardiaques de la grossesse et du post-partum sont rares mais potentiellement mortelles. Les diagnostics différentiels de la douleur thoracique en période de post-partum qui s'offrent alors à l'urgentologue comprennent souvent des causes graves telles que l'embolie pulmonaire ou l'infarctus du myocarde (IM). Autre cause moins connue d'IM mais non moins importante pour autant, la dissection spontanée de l'artère coronaire (DSAC), définie comme un hématome intramural de l'artère coronaire, qui obstrue la vraie lumière du vaisseau sanguin. Son extension provoquée par une élévation de la pression peut entraîner de l'ischémie myocardique, puis finalement un infarctus. Il s'agit de la cause la plus fréquente d'IM associée à la grossesse et elle explique de 24 à $35 \%$ des IM qui touchent les femmes de moins de 50 ans.
Le clivage de l'artère s'observe le plus souvent chez les jeunes femmes en bonne santé, qui n'ont pas de facteurs de risque classiques de coronaropathie ou de maladie cardiaque, et il se produit généralement durant le post-partum. La DSAC en phase de péripartum se définit comme la dissection spontanée de l'artère coronaire associée à la grossesse. Elle se manifeste par des altérations de l'électrocardiogramme, un taux élevé des troponines et des anomalies de la contractilité pariétale régionale à l'échocardiographie, tous des signes diagnostiques de la DSAC, que pourra finalement confirmer une coronarographie. Faute d'intervention immédiate, le clivage peut évoluer vers l'insuffisance cardiaque aiguë, le choc cardiogénique et la mort. Le traitement thrombolytique peut être dangereux et il n'est pas recommandé, et l'intervention coronarienne percutanée comporte un risque d'extension iatrogène de la dissection coronarienne. Aussi la prise en charge de la DSAC présumée consistet-elle en la mutation sans délai de la patiente dans un centre ou un service appropriés, et en une coronarographie d'urgence qui permettra de déterminer le meilleur traitement qui soit.

Keywords: spontaneous coronary artery dissection, chest pain, peripartum, female, pregnancy-associated spontaneous coronary artery dissection

\section{INTRODUCTION}

Cardiac emergencies in pregnancy and the postpartum period are rare but often life-threatening. An emergency physician's differential diagnosis for chest pain in the peripartum patient often includes serious etiologies such as pulmonary embolism or acute myocardial infarction (MI). A lesser-known but important consideration on the differential for $\mathrm{MI}$ is that of a spontaneous coronary artery dissection (SCAD). In pregnant women, SCAD is defined as pregnancyassociated spontaneous coronary artery dissection (PASCAD). This condition is the most common cause

From the ${ }^{*}$ Department of Family and Community Medicine and tDivision of Emergency Medicine, University of Toronto, Toronto, ON; $¥$ Assistant Director of Risk Management and Faculty Development, University Health Network, Toronto, ON; and the §Medical Director of Stadium Medicine, Toronto Blue Jays, Toronto, ON

Correspondence to: Dr. Richard Lee, Department of Family and Community Medicine, University of Toronto, 500 University Ave., Toronto, ON M5G 1V7; Email: Richard.Lee@uhn.ca 
of pregnancy-associated $\mathrm{MI}$ and is reported as the cause of $\mathrm{MI}$ in $24 \%$ to $35 \%$ of all women younger than 50 years. ${ }^{1,2}$ These patients may present to emergency rooms, yet this diagnosis receives limited attention among the emergency medicine community. ${ }^{3}$ Although PASCAD can be seen at any trimester in pregnancy and up to months postpartum, the majority of cases have been reported in the third trimester or early postpartum period defined as within 6 weeks of delivery. ${ }^{2,4}$ Abnormal electrocardiogram (ECG) changes, elevated troponins, and regional wall motional abnormalities on echocardiography are all diagnostic findings of PASCAD, which can be ultimately confirmed with coronary angiography. ${ }^{1,5}$ Failure to immediately address this condition can lead to acute heart failure, cardiogenic shock, and death. ${ }^{6}$

\section{CASE REPORT}

We describe a 33-year-old female who presented with shortness of breath and crushing central chest pain in the first 36 hours postpartum after her fifth uncomplicated spontaneous vaginal delivery of a healthy baby. She complained of nausea and shortness of breath and described 15 minutes of 10/10 crushing central chest pain without radiation.

On exam, she was afebrile with a heart rate of 81 , a blood pressure of $171 / 108$, and was saturating $100 \%$ on room air. On auscultation, there was good air entry bilaterally and normal heart sounds with no murmurs. No pain or edema was found in her lower extremities. Her ECG is shown in Figure 1. Her chest pain and shortness of breath improved after approximately 15 minutes with only mild retrosternal discomfort lasting a further 2 hours.

Laboratory results showed a hemoglobin of 87 and an initial troponin of $20 \mathrm{ng} / \mathrm{L}$ (reference range $<14 \mathrm{ng} / \mathrm{L}$ normal). Her repeat troponin 7 hours later was elevated at $1080 \mathrm{ng} / \mathrm{L}$, and she was treated with acetylsalicylic acid (ASA) $160 \mathrm{mg}$, clopidogrel $300 \mathrm{mg}$, and referred to a cardiologist.

Subsequent to her referral, she had a recurrent episode of chest pain lasting 30 minutes. Her ECG at this time revealed dynamic $0.5-\mathrm{mm}$ ST segment depressions in leads $\mathrm{V} 2-\mathrm{V} 3$ with $\mathrm{T}$-wave inversion in leads V2 (Figure 2). She was subsequently transferred to the Coronary Care Unit.

She received intermittent labetalol doses for blood pressure fluctuations and underwent cardiac catheterization. A coronary angiography revealed a decreased left anterior descending (LAD) flow consistent with a coronary artery aneurysm secondary to SCAD. She had a saccular aneurysm in the proximal LAD, 50\% occlusion in the mid LAD, and 20\% in the distal LAD. This was managed medically with blood pressure control (labetalol $200 \mathrm{mg}$ bid) and dual antiplatelet therapy

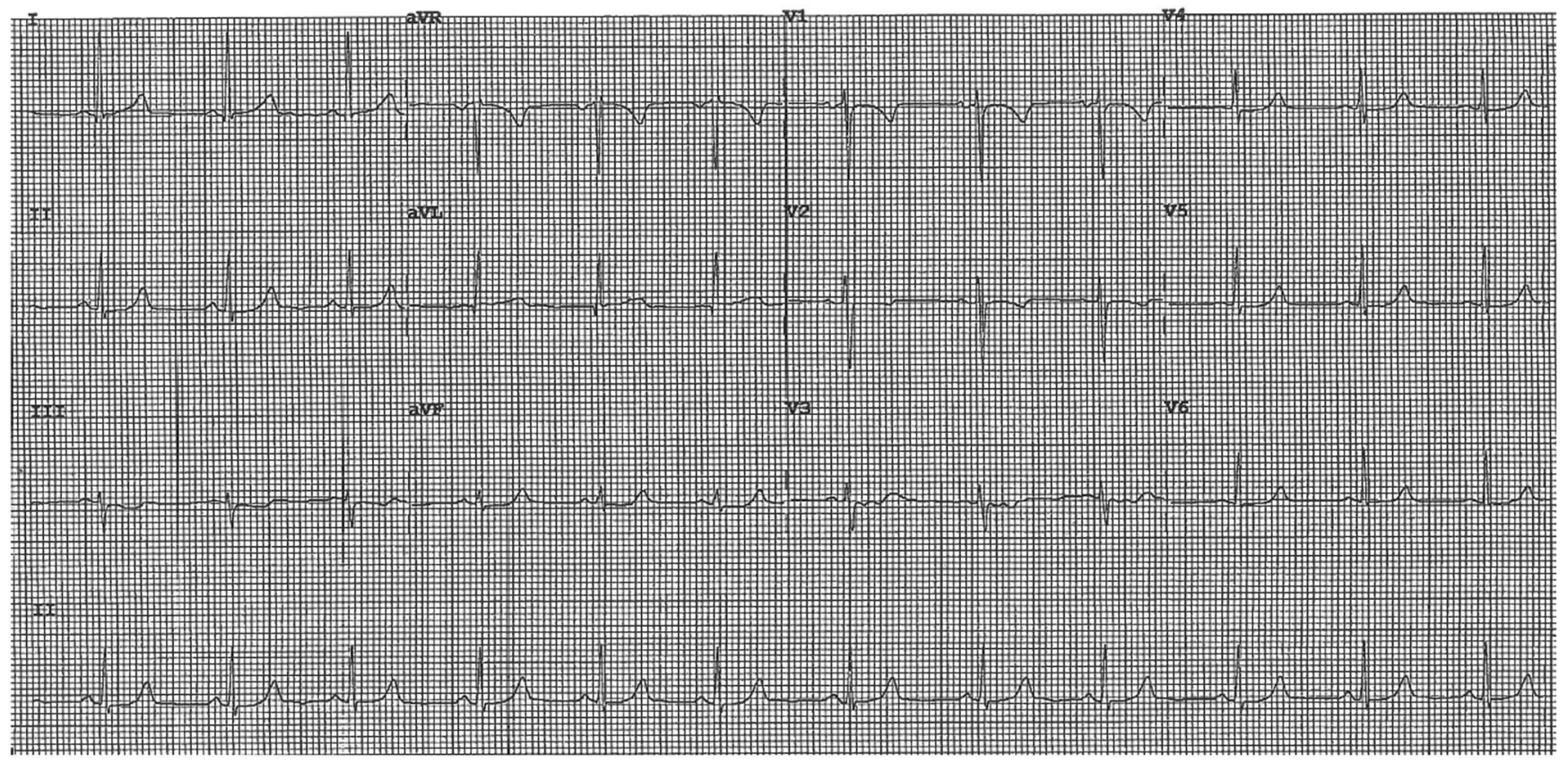

Figure 1. ECG on Presentation. 


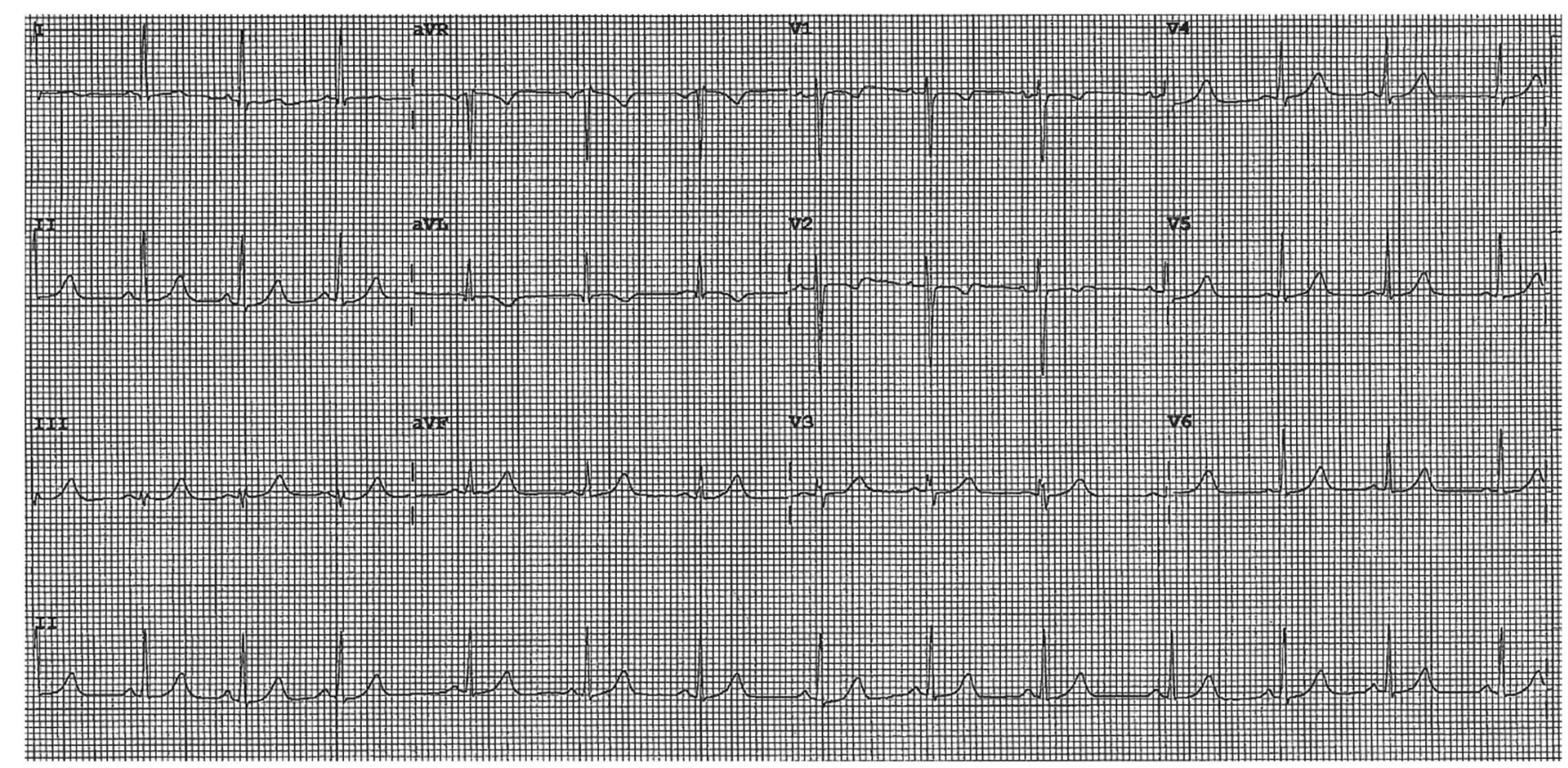

Figure 2. ECG Subsequent to Referral.

(ASA $81 \mathrm{mg}$, clopidogrel $75 \mathrm{mg}$ ) for 12 months. A bedside echocardiogram prior to discharge showed distal wall motion abnormalities in the distal inferoseptal area. The ejection fraction and valvular function were grossly normal, and there was no pericardial effusion present. She had no further episodes of chest pain and was asymptomatic at the time of discharge.

\section{DISCUSSION}

SCAD is considered a rare condition accounting for approximately $0.1 \%$ to $4 \%$ of all acute coronary syndrome (ACS) pathologies in the general population. ${ }^{7}$ Its wide clinical presentations range from being asymptomatic to the more typical angina to sudden cardiac death. SCAD is defined as an intramural hematoma within the coronary artery that compresses the true lumen and must be unrelated to trauma or medical instrumentation such as coronary catheterization. Expansion of this false lumen by increased pressures may lead to subsequent myocardial ischemia and infarction.

It is well known amongst clinicians that women often present with atypical features of ACS. Although this condition is a relatively infrequent cause of ACS in the general population, SCAD proportionally causes a higher number of MIs in young women., 8
Furthermore, acute MI is a significant cause of mortality and morbidity in peripartum women. ${ }^{4}$

Remarkably, SCAD has been described as accounting for $24 \%$ to $35 \%$ of $\mathrm{MI}$ in women under the age of 50 and is suspected to have an even higher prevalence in women with pregnancy-related $\mathrm{MI}^{2}{ }^{2}$ In pregnancy-related cases of MI, SCAD has been described as the most common mechanism and the underlying cause in $40 \%$ or more of cases. ${ }^{10}$ It has been identified as more common than atherosclerosis, thromboembolism, vasospasm, or Takotsubo syndrome in this population. Additionally, PASCAD is reported to have a higher acuity and worse prognosis than non-pregnancy-related SCAD cases. $^{2}$ Forty percent of PASCAD cases present as sudden death or die within hours of the onset of symptoms. ${ }^{9}$ However, those who survive this acute phase and receive proper diagnosis and treatment generally have a good prognosis. ${ }^{9}$

This condition is predominately seen in young healthy females with no traditional risk factors for coronary artery disease and typically in the postpartum period. ${ }^{1,11,12}$ Women account for approximately $82 \%$ to $90 \%$ of SCAD cases. ${ }^{8,13}$ SCAD has also been associated with fibromuscular dysplasia, connective tissue disease, and episodes of extreme emotion or exertion. ${ }^{1}$ Multiparity appears to be a risk factor, as does a previous history of SCAD. ${ }^{9}$ It is often seen at the end of pregnancy or early postpartum period where it is 
thought that the hormonal changes and hemodynamic stresses of pregnancy weaken the coronary artery wall and contribute towards its etiology. ${ }^{6}$

The wide clinical spectrum of PASCAD ranges from unstable angina to sudden cardiac death, but the primary presentation is often seen as non-ST elevation and STelevation MI. ${ }^{1}$ Classical signs and symptoms follow an ACS pattern and include chest pain, dyspnea, diaphoresis, and/or nausea. Initial diagnostic testing includes ECG and cardiac biomarkers. However, because these patients are often young and otherwise healthy, the diagnosis of PASCAD may be delayed or overlooked if repeat or serial testing is not considered. Therefore, clinicians must be aware of a potentially evolving ACS in this population and order serial ECGs and troponin levels to avoid missing or delaying a diagnosis of PASCAD. ${ }^{1}$ Abnormal ECG changes, elevated troponins, and regional wall motional abnormalities on echocardiography are all diagnostic findings of PASCAD, which can be ultimately confirmed with coronary angiography. 1,5

\section{MANAGEMENT}

Currently, no evidence-based management guidelines exist for SCAD or PASCAD due to its limited literature and lack of randomized control trials. ${ }^{4}$ Early intervention for these patients is imperative to allow for optimal outcomes. Blood pressure and heart rate control to limit vessel shearing is a critical part of the initial management. ${ }^{4}$ It appears unclear whether standard ACS pharmaceutical agents, such as ASA or clopidogrel, are indicated; yet patients often receive these initially because they may offer some protection and minimal harm. However, more potent agents, such as glycoprotein IIb/ IIIa inhibitors, are not routinely used because they carry a higher bleeding risk and potential for extending dissection. ${ }^{4,14}$ Additionally, the role of new P2Y12 antagonists, such as prasugrel and ticagrelor, is also unclear. ${ }^{14}$

The risks and benefits of anticoagulation in PASCAD are controversial. The risk of coronary artery dissection extension is met with potential resolution of a false lumen thrombus improving patency of the true lumen. Heparin agents started empirically have been discontinued once coronary artery dissection is declared on angiography to avoid the worsening of intramural hematoma. ${ }^{4,14}$ Moreover, thrombolytic treatment in PASCAD can be harmful and is not recommended because it may expand the dissection and worsen coronary vasospasm. ${ }^{4,12}$ As a result, when PASCAD is highly suspected, urgent coronary angiography is crucial because PASCAD medical management differs from standard ACS therapy. ${ }^{14}$ However, in remote centres without routine access to primary percutaneous coronary intervention (PCI), thrombolysis may need to be considered for ST-elevation MI patients. ${ }^{14}$ Yet, because PASCAD has been described as the most common mechanism of pregnancy-associated $\mathrm{MI}$ and documented in over $40 \%$ of cases, thrombolytic therapy should only be considered after the risks and benefits have been assessed and when little other option exists in an unstable patient (Figure 3). In an analysis of 120 PASCAD cases by Havakuk et al., thrombolytic therapy was used in 10 cases and followed by either PCI (3 cases) or coronary artery bypass grafting (CABG) $\left(5\right.$ cases). ${ }^{10}$

When ACS is recognized in a peripartum patient, urgent coronary artery catheterization should be performed to confirm a diagnosis of PASCAD and determine appropriate therapeutic strategies for management. ${ }^{1,4,5}$ Urgent angiography is often required for both the diagnosis of PASCAD and to rule out acute plaque occlusion. Emergency physicians need to have an early suspicion for potential PASCAD in female patients presenting with ACS symptoms and arrange for urgent coronary artery catheterization or a prompt transfer to a centre where this capability exists.

Treatment strategies are decided based on the extent and character of dissection detected on angiography and can include medical therapy, stenting for revascularization, or CABG. Hemodynamically stable patients with a single-vessel disease without progression, without a left main coronary artery involvement or danger to a large area of myocardium may be medically managed with close observation. ${ }^{9}$ In contrast to atherosclerotic disease, PCI is associated with elevated rates of complications, including the risk of further dissection or subsequent emergency CABG. ${ }^{1}$ Complications with PCI can occur even in cases where there has been normal or near-normal coronary artery blood flow. ${ }^{1,4}$ Thus, PCI or coronary stenting is not typically performed.

\section{CONCLUSION}

Chest pain or an ACS equivalent in a young peripartum woman without traditional cardiac risk factors should prompt an emergency physician to consider PASCAD.,12 This diagnosis is often overlooked and needs to be added to the list of serious etiologies for chest pain in a young 


\section{Approach for suspected SCAD in a peripartum patient}

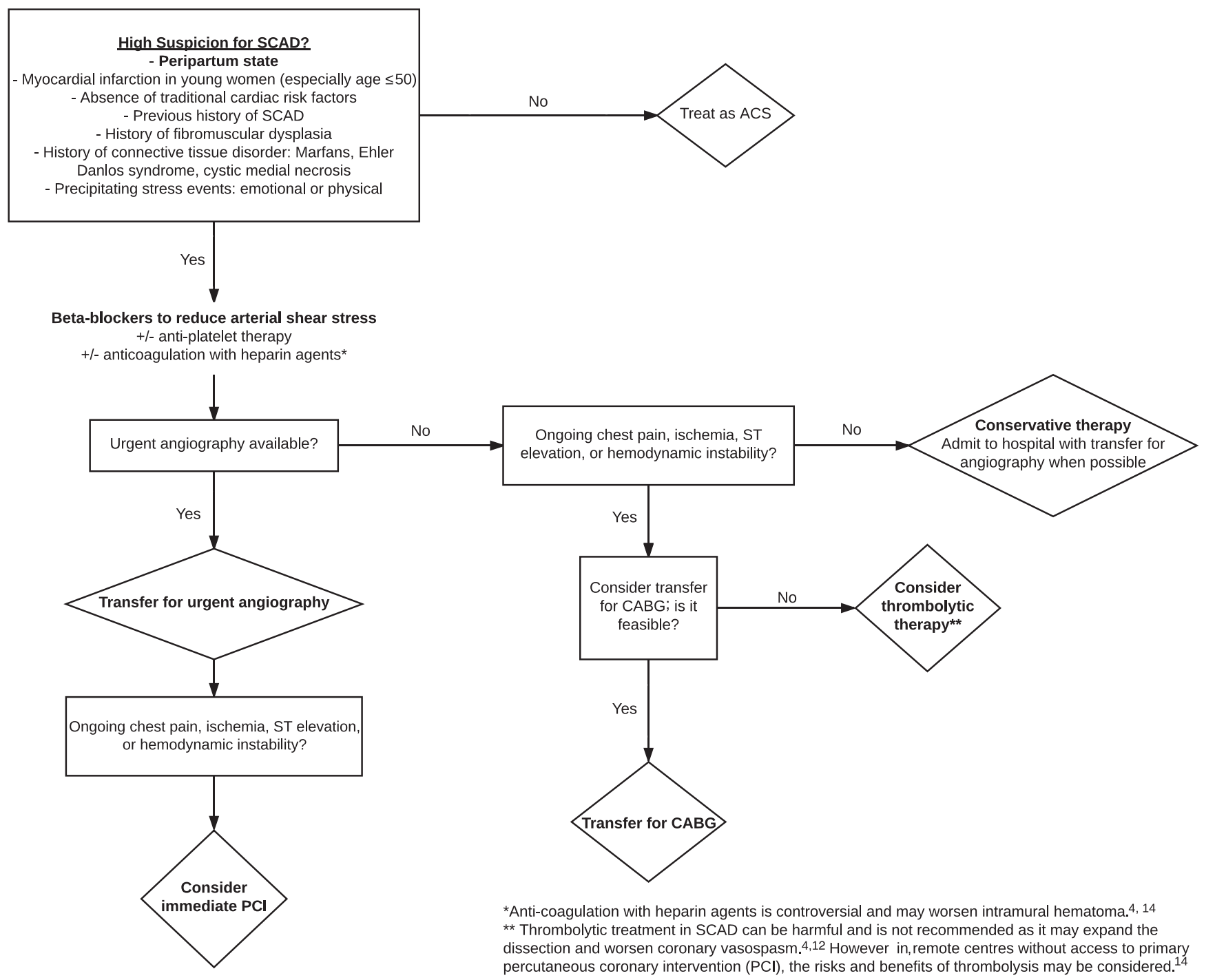

Figure 3. An Approach to PASCAD.

patient such as pulmonary embolism, MI, or aortic dissection. SCAD is an important cause of ACS in women, accounting for $24 \%$ to $35 \%$ of $\mathrm{MI}$ in women under the age of $50 .{ }^{2,8}$ Furthermore, PASCAD has been described as the most common mechanism of pregnancyassociated MI and documented in over $40 \%$ of cases. ${ }^{10}$ Rather than predominately thinking about pulmonary embolism in this population, one needs to add PASCAD to the differential diagnosis.

Immediate blood pressure and heart rate control are necessary to limit vessel sheering until urgent coronary angiography can be performed to confirm the diagnosis. Prompt arrangements for urgent coronary catheterization need to be made, because it is crucial to the diagnosis and to help avoid potentially harmful treatments. When compared with atherosclerotic disease, unique concerns exist in PASCAD. Thrombolytic treatment may be harmful and is not recommended, and PCI can result in the iatrogenic propagation of further coronary dissection. ${ }^{4,15}$

\section{TAKE HOME POINTS}

"Take Home" clinical messages for emergency physicians include the following:

1) SCAD as a cause of MI should be on the differential diagnosis of serious etiologies for chest pain in a 
young patient (i.e., pulmonary embolism/ACS/ aortic dissection/SCAD).

2) Chest pain in a young peripartum female without traditional cardiac risk factors should prompt an emergency physician to think beyond pulmonary embolism and consider MI, which includes PASCAD in the differential.

3) Management of suspected PASCAD: Because anticoagulation with heparin is controversial with the risk of worsening intramural hematoma, thrombolytics also potentially harmful and currently not recommended, the emergency management of suspected PASCAD needs to place emphasis on prompt recognition and an urgent transfer to a primary angiography centre for coronary artery catheterization. This allows an individual risk benefit decision on the best treatment strategy by vascular or cardiac surgeons and cardiologists. ${ }^{4,15}$

Competing interests: None declared.

\section{REFERENCES}

1. Tweet MS, Gulati R, Hayes SN. What clinicians should know about spontaneous coronary artery dissection. Mayo Clin Proc 2015;90(8):1125-30.

2. Saw J. Pregnancy-associated spontaneous coronary artery dissection represents an exceptionally high-risk spontaneous coronary artery dissection cohort. Circ Cardiovasc Interv 2017;10(3):e005119.

3. Ghaffarian K, Furin M, Bassett R. STEMI in a young female? Consider spontaneous peripartum coronary artery dissection. Am 7 Emerg Med 2017;35(2):381.e1-4.
4. Rose E, Gedela M, Miller N, Carpenter PL. Pregnancyrelated spontaneous coronary artery dissection: a case series and literature review. 7 Emerg Med 2017; 52(6):867-74.

5. Aliyary S, Mariani MA, Verhorst PM, et al. Staged therapeutic approach in spontaneous coronary dissection. Ann Thorac Surg 2007;83(5):1879-81.

6. Goland S, Schwarz ER, Siegel RJ, Czer LS. Pregnancyassociated spontaneous coronary artery dissection. Am $\mathcal{J}$ Obstet Gynecol 2007;197(6):e11-3.

7. Saw J. Spontaneous coronary artery dissection. Can 7 Cardiol 2013;29(9):1027-33.

8. Saw J, Aymong E, Mancini GJ, et al. Non-atherosclerotic coronary artery disease in young women. Can 7 Cardiol 2014;30(7):814-9.

9. Marcoff L, Popescu A, Leidig GA, et al. Spontaneous coronary artery dissection in a postpartum woman presenting with chest pain. Am 7 Emerg Med 2010;28(5):641.e5-e7.

10. Havakuk O, Goland S, Mehra A, Elkayam U. Pregnancy and the risk of spontaneous coronary artery dissection. Circ Cardiovasc Interv 2017;10(3): doi:10.1161/circinterventions.117.004941.

11. Teruzzi G, Calligaris G, Ravagnani P, et al. Peripartum spontaneous coronary artery dissection: a case report. Giornale Italiano di Cardiologia 2016;17(10):24S-7S.

12. Pabla JS, John L, Mccrea WA. Spontaneous coronary artery dissection as a cause of sudden cardiac death in the peripartum period. BMF Case Rep 2010, doi:10.1136/ bcr.05.2010.2994.

13. Tweet MS, Hayes SN, Pitta SR, et al. Clinical features, management, and prognosis of spontaneous coronary artery dissection. Circulation 2012;126(5):579-88.

14. Yip A, Saw J. Spontaneous coronary artery dissection a review. Cardiovasc Diagn Ther 2015;5:37-48, doi:10.3978/j. issn.2223-3652.2015.01.08.

15. Movahedian M, Leon M, Sibai B, Moussa H. Acute myocardial infarction due to coronary artery dissection in the postpartum period. Am 7 Perinatol Rep 2015;5(2): e093-6. 